\title{
Toxicity Assessment upon Thermal Treatments of Chlorpyrifos and 3,5,6-Trichloro-2-Pyridinol (a Potential Precursor of the Pyridine Analogue of 2,3,7,8-T4CDD) Using the Early Developmental Stage Medaka Embryos (Oryzias latipes)
}

\author{
Katsumasa HANNO*, Shoji ODA**, Takeshi NAKANO***, Hiroshi MITANI** \\ *Chiba Prefectural Environmental Research Center, Chiba 290-0046, Japan \\ **Department of Integrated Biosciences, Graduate School of Frontier Sciences, The University \\ of Tokyo, Chiba 277-8562, Japan \\ ***Graduate School of Engineering, Osaka University, Osaka 565-0871, Japan
}

\begin{abstract}
Chlorpyrifos is one of the major pesticides used today which has a chlorinated aromatic moiety of a pyridine analogue of 2,4,5-trichlorophenol and can be a potential direct precursor of another pyridine analogue of 2,3,7,8-T4CDD (2,3,7,8-tetrachlorodibenzo- $p$-dioxin). In the recent study, PCDF compounds have been detected in chlorpyrifos samples, however, the presence of this analogue of 2,3,7,8-T4CDD has not been assessed yet to our knowledge. Here, we analyzed the ecotoxicities of this dioxin-like compound in the early developmental stage embryos (Oryzias latipes). Chlorpyrifos and its analogue, 3,5,6-trichloro-2-pyridinol, were thermally treated in closed glass ampoules at 300,340 , and $380^{\circ} \mathrm{C}$ for 10 minutes and exposed to medaka embryos in early developmental stage for 48 hours, to assess the response of the 20 biomarker gene expressions. The thermally treated 3,5,6-trichloro-2-pyridinol enhanced the expression of CYP1A1 (Cytochrome P450 1A1) and AhRR (aryl hydrocarbon receptor repressor) as 2,3,7,8-T4CDD and PCBs, suggesting that the potentially ecotoxic compounds like 2,3,7,8-T4CDD is newly produced during the thermal treatment of 3,5,6-trichloro-2-pyridinol. The expression profiles of the 20 biomarker genes in the embryos exposed to 2,3,7,8-T4CDD and 3,5,6-trichloro-2-pyridinol were almost the same, strongly suggesting that the thermally treated 3,5,6-trichloro-2-pyridinol sample contained 2,3,7,8-T4CDD-like ecotoxic compounds.
\end{abstract}

Keywords: chlorpyrifos, medaka embryos, pyridinol, thermal treatment

\section{INTRODUCTION}

Pesticide production, use and disposal have contributed significantly to polychlorinated dibenzo-p-dioxins (PCDD) and polychlorinated dibenzofuran (PCDF) emissions in the past (Masunaga et al., 2001; Weber et al., 2008). In a recent monitoring of currently used pesticides in Australia in all assessed formulations, PCDD and PCDF were detected at high concentrations in some pesticide formulations (Holt et al., 2010). From a historic perspective, the two pesticides with the highest dioxin production and release were 2,4,5-trichlorophenoxy acetic acid (2,4,5-T) and pentachlorophenol (PCP) (Hagenmaier and Berchtold, 1986; Weber et al., 2008). The production and use of 2,4,5-T and 2,4,5-trichlorophenol, which can be a precursor of 2,3,7,8-T4CDD, resulted in large contamination during the Vietnam War (366 kg TEQ (toxicity equivalency quantity) release from Agent Orange spray) and had a long series of dioxin contamination in the factories with the last accident in Seveso where about $30 \mathrm{~kg}$ TEQ have been released (Mocarelli 2001). Today, 2,4,5-T is not available in the market and the use of 2,4,5-trichlorophenol is restricted to the synthesis of the still available

Address correspondence to Katsumasa Hanno, Chiba Prefectural Environmental Research Center, Email: k.hnn@pref.chiba.lg.jp

Received, May 12, 2012, Accepted, February 28, 2013. 
hexachlorophene, one of the major pesticides used today. Another major pesticide used today, chlorpyrifos, has a chlorinated aromatic moiety of a pyridine analogue, 2,4,5-trichlorophenol (3,5,6-trichloro-2-pyridinol; pyridinol), making this pesticide to be a potential direct precursor of the other pyridine analogue, 2,3,7,8-T4CDD. In the recent study, PCDFs have been detected in chlorpyrifos samples and the presence of chlorpyrifos has been assessed with GC/MS (gas chromatograph/mass spectrometry) analysis by Sakiyama et al. (2011). Waste incineration facilities in Japan today are advised to incinerate the wastes at $850^{\circ} \mathrm{C}$ or higher, so that the pyrolysates of thermally treated pyridinol or chlorpyrifos would be completely decomposed and detoxified. However, inside the waste incinerator significant variation in temperature can occur and we can estimate that dioxins and dioxin-like compounds (e.g. the pyridine analogue of 2,3,7,8-T4CDD) might be formed in the regions at low temperature (e.g. $300-380^{\circ} \mathrm{C}$ ) in the waste incinerator or in the case of illegal incineration (e.g. "open burning", "NOYAKI"). In this study, we analyzed the ecotoxicities of these dioxin-like compounds in the early developmental stage embryos of medaka (Oryzias latipes). Chlorpyrifos and pyridinol were thermally treated to form their pyrolysates at moderate temperatures and their precursor potentials were addressed. Medaka embryos in early developmental stage were exposed to these pyrolysates for 48 hours and the ecotoxicogenomics response were assessed by the changes in expressions of the 20 biomarker genes specifically chosen for the evaluation of the ecotoxicities of environmental water.

\section{MATERIALS AND METHODS \\ Thermal treatment}

Chlorpyrifos and 3,5,6-trichloro-2-pyridinol (pyridinol) were purchased from Wako Pure Chemicals (chemical standard grade, Japan). All pyrolysis experiments were carried out in sealed brown glass ampoules; $0.2 \mathrm{mg}$ of chlorpyrifos or $0.8 \mathrm{mg}$ of pyridinol were thermally treated in closed brown glass ampoules at 300,340 , and $380^{\circ} \mathrm{C}$ for 10 minutes in a GC-oven (Agilent, USA). After cooling down to room temperature, the ampoules were opened carefully. Then, the pyrolysate products were extracted with toluene (pesticide grade; Wako Pure Chemicals) and the toluene was evaporated to the volume of $1.5 \mathrm{~mL}$ under gentle nitrogen stream. One hundred and fifty $\mu \mathrm{L}$ of the extracts dissolved in toluene was placed in a vial to be dried up and the pyrolysates were redissolved in DMSO $60 \mu \mathrm{L}$. 3,3'-dichlorobiphenyl (PCB11), 3,3',4,4',5-pentachlorobiphenyl (PCB126) and 2,3,7,8-T4CDD were purchased from Wellington Laboratories (Ontario, Canada) in a nonane solution, evaporated, and then redissolved in DMSO at a concentration of $5 \mu \mathrm{g} / \mathrm{L}$.

\section{Medaka embryos and exposure to waterborne chlorpyrifos}

Fertilized eggs and embryos were obtained from natural mating of adult orange-red strain medaka fish originally derived from the colony kept in The University of Tokyo and maintained at $26 \pm 0.5^{\circ} \mathrm{C}$ under a 14 hours-light and 10 hours-dark cycle. Newly fertilized eggs ( $200-300$ embryos) were placed in a $100 \mathrm{~mL}$ beaker with distilled water and incubated for 24 hours at $26 \pm 0.5^{\circ} \mathrm{C}$ to develop. It is noteworthy that in this study the medaka embryos at 25 hours post-fertilization (hpf) were exposed to chlorpyrifos and pyridinol in the early neurula stage (Stage17) to eliminate abnormally developing or dead embryos within the first 24 hours of development. We settled the live embryos 
(Stage17) into wells of microtiter plates filled with $200 \mu \mathrm{L}$ of waterborne vehicle (DMSO) with $0.32 \mathrm{mg} / \mathrm{L}$ of chlorpyrifos, $1.3 \mathrm{mg} / \mathrm{L}$ of pyridinol and $1 \mu \mathrm{g} / \mathrm{L}$ of dioxins (PCB11, PCB126 and 2,3,7,8-T4CDD). The final concentration of the vehicle (DMSO) was to be $0.1 \%(\mathrm{v} / \mathrm{v})$. In these studies, 3 days post-hatch was considered to represent survival, and we confirmed that the distilled water and DMSO in the range of $0-0.2 \%$ $(\mathrm{v} / \mathrm{v})$ did not induce any abnormalities in the early developmental stage of medaka embryos.

\section{Total RNA isolation and real-time PCR experiments}

Total RNA was isolated from the exposed embryos by ISOGEN (Nippon Gene, Japan) and then purified with RNeasy Mini Kit (QIAGEN, USA). The quality and quantity of isolated total RNA were verified by agarose gel electrophoresis and a spectrophotometer (ND-1000, Nano Drop Tech., USA). Total RNA (400 - 1,000 ng/ $\mu \mathrm{L})$ with an OD260/ OD280 ratio over 2.0 were obtained from $30-50$ embryos and used. Complementary DNAs (cDNAs) were synthesized from $1 \mu \mathrm{g}$ of total RNA using Rever TraAce- $\alpha^{\mathrm{TM}}$ (Toyobo, Japan) and oligo(dT)20 primers (Takara, Japan). The relative amounts of the transcripts were quantified using real-time PCR which was performed using SYBR ${ }^{\circledR}$ Premix Ex Taq ${ }^{\mathrm{TM}}$ in a Smart Cycler ${ }^{\circledR}$ II System (Takara, Japan) and specific primers, according to the manufacturer's instructions. For each sample, gene expression was analyzed in triplicate with the following protocol: $95^{\circ} \mathrm{C}$ for $10 \mathrm{~s}$, followed by 45 cycles of $95^{\circ} \mathrm{C}$ for $5 \mathrm{~s}$ and $60^{\circ} \mathrm{C}$ for $20 \mathrm{~s}$. At the completion of each PCR run, the obtained PCR products were subjected to melting curve analysis to ensure that only a single product was amplified. At least three technical replicates of each RNA sample were conducted. Expression data were quantified based on threshold cycle $(\mathrm{Ct})$ values. For each gene, the $\mathrm{Ct}$ values for each sample were averaged and normalized to the mean $\mathrm{Ct}$ of EF-1 $\alpha$ (elongation factor 1-alpha), which is expressed ubiquitously in tissues and widely used as gene expression control. Relative mRNA expression for each gene was calculated as the fold change compared with the solvent control, exposed to DMSO $(0.1 \%(\mathrm{v} / \mathrm{v}))$ for $48 \mathrm{~h}$, according to equations (1) and (2) (Livak and Schmittgen, 2001; Hanno et al., 2010) :

$$
\begin{aligned}
& \text { Relative mRNA expression }=2^{-\Delta \Delta \mathrm{Ct}} \\
& \Delta \Delta \mathrm{Ct}=\left[\mathrm{Ct}_{\text {(target samples) }}-\mathrm{Ct}_{(\mathrm{EF}-1 \alpha)}\right]_{\text {exposed } 48 \mathrm{~h}}-\left[\mathrm{Ct}_{(\text {solvent control })}-\mathrm{Ct}_{(\mathrm{EF}-1 \alpha)}\right] \text { exposed } 48 \mathrm{~h}
\end{aligned}
$$

\section{Medaka-customized microarray experiments.}

Medaka-customized microarray (Agilent, USA) contains 36,398 medaka gene probes. Following hybridization at $65^{\circ} \mathrm{C}$ for 17 hours, $10 \%$ Triton $\mathrm{X}-100$ was added to the array to make a final concentration of $0.005 \%$ and washed with Wash Bufferl and Wash Buffer2. Fluorescence signals were scanned using Agilent DNA Microarray Scanner. All microarray data were quantified using Feature Extraction Software (Agilent, USA).

\section{Statistical analysis}

Statistical significance was determined by the $t$-test. The results were presented as fold difference between the chemicals treated groups and the control groups. 


\section{RESULTS AND DISCUSSION}

We classified the biomarker genes into four groups, using a medaka-customized microarray: Group1: CYP1A1, UDPGT (uridine diphosphate glucuronyl transferase), AhR1b-1, AhR2a, AhRR, ER- $\beta$ (estrogen receptor-beta) - detoxication metabolism genes; Group2: CACHD1 (Cache Domain containing 1), $R A R-\alpha$ (retinoic acid receptor-alpha), $E R-\alpha, V E G F-R$ (vascular endothelial growth factor receptor) endocrine effect/reproduction genes; Group3: $A G X T$ (alanine-glyoxylate aminotransferase), MTF1 (metal-regulatory transcription factor 1), Tropomyosin, HSP90 (heat shock protein 90) - cell proliferation genes; and Group4: TBP (TATA-box binding protein), $T N F-R$ (tumor necrosis factor receptor), $C D C 37$ (cell division control protein 37), HSP70, MT (metallothionein), ependymin 1 - immunity effect/nervous system genes, considering the physiological functions and induction by chemicals. In particular, Groupl genes were highly inducible by dioxins and dioxin-like compounds exposure. Induced expressions of the biomarker genes mRNA are shown in Table 1 . The shaded boxes represent the relative mRNA expression which showed up-regulated $(>2$-fold) or down-regulated $(<0.5$-fold) and significant difference at $\mathrm{p}<0.001(* * *)$ from the corresponding control value.

\section{Group1 genes}

The functions of the Group1 genes were detoxication and metabolism. CYP1A1 and $A h R R$ genes are well known to be highly inducible by dioxin and dioxin-like compounds exposure. Both genes are regulated through the aryl hydrocarbon receptor (AhR)-mediated pathway, which is ubiquitously functional in both mammalian tissues and medaka (Oryzias latipes) embryos. Although pyridinol blank did not induce the CYP1A1 expression, thermal treated pyridinol with higher temperature at $300-380^{\circ} \mathrm{C}$ induced higher expression of CYPIAl in medaka embryos (Fig. 1). In contrast, chlorpyrifos didn't enhance CYP1A1 expression with thermal treatment. The relative expression of CYPIAl was highly related to the WHO-TEF (World Health Organization - Toxic Equivalency Factor) for mammals (Hanno et al., 2010) and $380^{\circ} \mathrm{C}$ thermally treated pyridinol induced CYPIAl expression as dioxins or dioxin-like PCBs did.

The induced expression of $A h R R$ mRNA is shown in Fig. 2. In the adult killfish, $A h R R$ mRNA is widely expressed in tissues and is induced by both 2,3,7,8-T4CDD or dioxin-like PCBs (Hahn et al., 2009). AhRR might play significant roles in modulating the activities of $A h R s$ and mediating the induction of CYPlAl expression in fish as in mammals. In this study, AhRR expression was enhanced $2.0-2.5$ fold higher than the control upon exposure to $380^{\circ} \mathrm{C}$ thermally treated pyridinol, which was very similar when exposed to PCB-126 $(1 \mu \mathrm{g} / \mathrm{L})$ and 2,3,7,8-T4CDD $(1 \mu \mathrm{g} / \mathrm{L})$. Four $A h R$ genes (AhR1b-1,AhR1b-2, AhR2a and AhR2b) were identified in the medaka genome and $A h R 2 a$ is most strongly induced by $2,3,7,8-T 4 C D D$, which suggests that $A h R 2 a$ plays an important role as dioxins receptors in meditating the induction of CYPIA1 and the ecotoxicities of dioxins in medaka embryos (Hanno et al., 2010).

In contrast, $A h R 1 b-1$ was only slightly induced by $2,3,7,8$-T4CDD and endogenous expression increased during normal development in unexposed embryos, suggesting that $A h R 1 b-1$ possesses physiological functions distinct from the dioxin response in medaka (Hanno et al., 2010). Induction of $A h R 2 a$ mRNA expression is shown in Fig. 3. 


\begin{tabular}{|c|c|c|c|c|c|c|c|c|c|c|c|c|c|c|c|c|c|c|c|c|c|c|c|}
\hline$=$ & $*$ & * & $*$ & $*$ & ** & * & & $\frac{5}{2}$ & 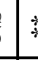 & & 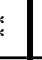 & $*$ & $z$ & $*$ & $*$ & $*$ & * & * & 1 & $*$ & 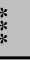 & & $\dot{m} \stackrel{n}{*} v$ \\
\hline \begin{tabular}{l|}
$\mathscr{D}$ \\
$m_{1}$ \\
$\bar{z}$
\end{tabular} & है: & 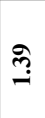 & 象 & $\mid \begin{array}{l}\text { t } \\
0 \\
0 \\
0\end{array}$ & 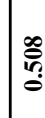 & 离 & $\stackrel{2}{g}$ & 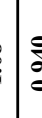 & 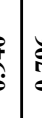 & & : & $\stackrel{\mathscr{m}}{\rightarrow}$ & 変 & $\overline{\underline{s}}$ & $\underset{I}{\stackrel{T}{g}}$ & 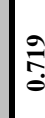 & $\approx$ & 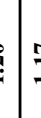 & & ְ̦ & đֶ. & $\stackrel{\substack{\infty \\
\infty}}{\infty}$ & 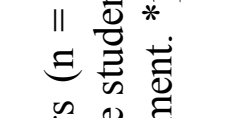 \\
\hline$=$ & $\frac{n}{z}$ & $*$ & $\frac{n}{z}$ & 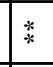 & 年 & $*$ & * & * & 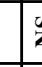 & 8 & & *. & $*$ & $*$ & $*$ & * & * & $:$ & 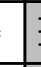 & $*$ & 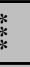 & $*$ & 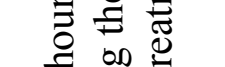 \\
\hline $\begin{array}{l}\text { 导 } \\
\vec{m}_{1} \\
\vec{z}^{\prime}\end{array}$ & $\stackrel{3}{-}$ & สี & $\exists$ & $\stackrel{\infty}{\stackrel{\infty}{3}}$ & : & $\stackrel{m}{2}$ & 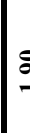 & 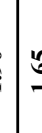 & 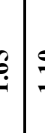 & 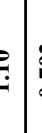 & 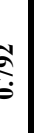 & : & $\dddot{ְ}$ & F & हn & 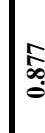 & 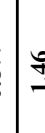 & & & 商 & ج্: & $\stackrel{0}{=}$ & 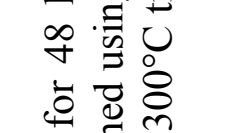 \\
\hline$=$ & $\frac{n}{z}$ & $*$ & $z$ & 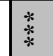 & $\frac{\pi}{2}$ & $z$ & $*$ & $*$ & 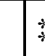 & & " & 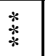 & $*$ & $*$ & $*$ & in & $*$ & 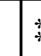 & 1 & $*$ & 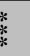 & 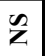 & $\overline{0}$ \\
\hline \begin{tabular}{l|}
$\tilde{e}_{1}$ \\
$\vec{z}^{\prime}$
\end{tabular} & 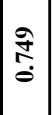 & $\stackrel{\Xi}{-}$ & ô. & î & a & $\stackrel{9}{ت}$ & 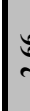 & 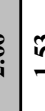 & 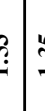 & $?$ & 点 & $\stackrel{F}{ت}$ & ঙ্গ & ing & : & ठี̃ & 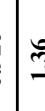 & & & | & స̂ & $\Xi$ & 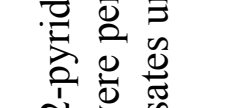 \\
\hline$=$ & $*$ & $\stackrel{*}{*}$ & $*$ & 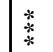 & 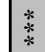 & $z$ & * & $*$ & 1 & 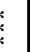 & 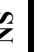 & * & $\stackrel{4}{*}$ & $*$ & $*$ & $*$ & $*$ & 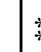 & 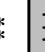 & 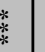 & * & $*$ & 07 \\
\hline 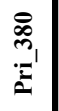 & $\approx$ & $\overline{\vec{i}}$ & 苛 & 象 & $\sqrt[3]{\pi}$ & $\dddot{9}$ & 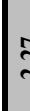 & 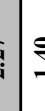 & 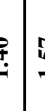 & 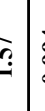 & S. & $\overrightarrow{3}$ & $\stackrel{\infty}{\dddot{\leftrightarrow}}$ & $\tilde{\overbrace{}}$ & 管 & $\mid \begin{array}{l}\infty \\
0 \\
\dot{0}\end{array}$ & $=$ & $\vdots$ & 3 & 亭 & क़ & 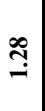 & 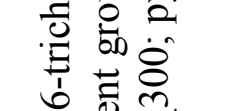 \\
\hline$=$ & $*$ & $\frac{n}{z}$ & $*$ & $\frac{n}{2}$ & * & * & $*$ & * & . & & $*$ & 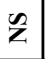 & 站 & $*$ & * & $\%$ & 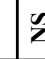 & \% & 负 & 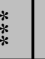 & $*$ & $*$ & $m$ \\
\hline 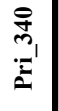 & की & $\begin{array}{l}\infty \\
0 \\
0 \\
0\end{array}$ & \begin{tabular}{|l|l} 
\\
0 \\
0 \\
0 \\
0
\end{tabular} & 告 & 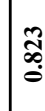 & 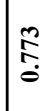 & 2 & 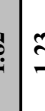 & 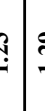 & בे & 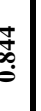 & $\stackrel{\Xi}{=}$ & $\stackrel{\leftrightarrow}{-}$ & ণิ & : & $\stackrel{\hat{n}}{0}$ & $\cong$ & & : & ?. & $\frac{8}{6}$ & 苞 & 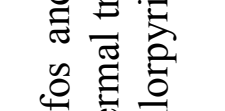 \\
\hline$=$ & $*$ & $*$ & $\frac{n}{z}$ & $\frac{\alpha}{z}$ & $*$ & $*$ & * & $\frac{5}{2}+a x$ & \& & E & $*$ & $*$ & $*$ & $*$ & * & $y^{n}$ & ?. & $:$ & 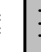 & 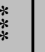 & 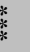 & * & \\
\hline $\begin{array}{l}D_{n} \\
m_{1} \\
\vdots \\
\vdots\end{array}$ & $\underset{j}{\infty}$ & त્ & $\stackrel{\Xi}{-}$ & $\stackrel{\Xi}{=}$ & 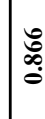 & $\stackrel{\overbrace{}}{\mathscr{T}}$ & $i$ & $\frac{4}{7}$ & 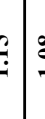 & 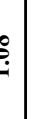 & 萬 & 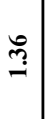 & $\stackrel{\overbrace{}}{\dddot{m}}$ & Fi & : & $\stackrel{\Xi}{i}$ & 9 & 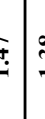 & $\infty$ & 童 & 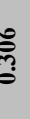 & $\overline{3}$ & 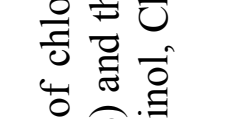 \\
\hline$=$ & $\frac{n}{z}$ & * & $*$ & * & * & $*$ & . & * & 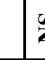 & $\hat{z}$ & $*$ & $\frac{n}{z}$ & z & $*$ & $*$ & $*$ & $z$ & $\theta$ & 安 & * & 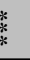 & z & $\theta \circ 0$ \\
\hline$\vec{m}$ & 离 & $\stackrel{\infty}{=}$ & శ్ర్: & 柋 & 常 & $\stackrel{8}{2}$ & $\tilde{9}$ & $\bar{\alpha}$ & לִ & 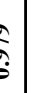 & \begin{tabular}{l}
0 \\
0 \\
\hdashline \\
\hdashline
\end{tabular} & ลั & 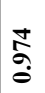 & $\stackrel{\text { fof }}{-1}$ & |⿺辶大: & t: & : & & $e^{2}$ & 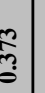 & $\frac{8}{6}$ & ֻू̆ & \\
\hline$=$ & : & $\underline{z}$ & $*$ & $*$ & 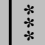 & $*$ & $z$ & $*$ & 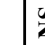 & 8 & 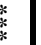 & $*$ & $*$ & $*$ & * & * & : & : & : & $n$ & * & $*$ & \\
\hline 10 & $\approx$ & $\stackrel{\square}{\leftrightarrows}$ & $\dddot{\ddots}$ & 赵 & ì & 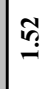 & 8 & $\because$ & 9 & 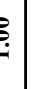 & 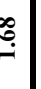 & $\stackrel{\infty}{-\infty}$ & 苞 & $\stackrel{3}{3}$ & $\underset{\infty}{\tilde{\infty}}$ & $\stackrel{\sharp}{I}$ & 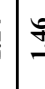 & 里 & 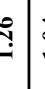 & $\stackrel{\square}{-}$ & : & $\stackrel{t}{\rightarrow}$ & 氖: \\
\hline$=$ & $*$ & $*$ & $*$ & $*$ & $*$ & $\frac{n}{2}$ & $\frac{5}{2}+2 \cdot a+2$ & $*$ & 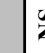 & \& & $n$ & $\frac{n}{z}$ & z & $*$ & $*$ & $*$ & s & : & 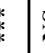 & $\frac{n}{2}$ & 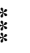 & $*$ & a \\
\hline 䜦 & $\stackrel{\rho}{\dot{p}}$ & 苛 & 甯 & 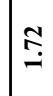 & $\underset{\mathrm{i}}{\stackrel{t}{\mathrm{i}}}$ & $\stackrel{7}{=}$ & $\stackrel{a}{g}$ & 2 & : & . & : & $\fallingdotseq$ & Dू. & ণ্ডి & 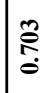 & 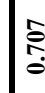 & $\tilde{g}$ & $\$$ & 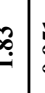 & S. & 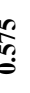 & กุ & 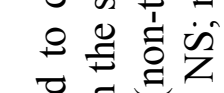 \\
\hline$=$ & $*$ & $\sum_{z}^{n}$ & $\frac{n}{2}$ & $*$ & $*$ & 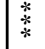 & $*$ & 菜 & 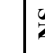 & \& & $*$ & $*$ & $*$ & $\stackrel{*}{*}$ & $\frac{n}{2}$ & $*$ & * & 1 &. & * & 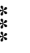 & $*$ & : \\
\hline 产 & $\stackrel{9}{-}$ & :ี & $\fallingdotseq$ & ఫิ & $\stackrel{\infty}{3}$ & 곡 & 5 & 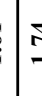 & 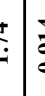 & 5 & ?: & $\underset{\mathrm{i}}{\stackrel{\mathrm{i}}{\mathrm{s}}}$ & 皇 & : & $\stackrel{\Xi}{\leftrightarrows}$ & $\stackrel{4}{\dddot{9}}$ & 5 & 5 & تَ & 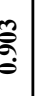 & 苞 & 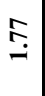 & $\begin{array}{lll}0 & 1 \\
0 & 0 \\
0 & 0 \\
0 & 0 \\
z & 0\end{array}$ \\
\hline 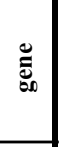 & 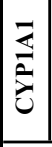 & $\begin{array}{l}\hat{0} \\
\hat{\vdots}\end{array}$ & 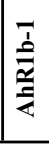 & 踏 & 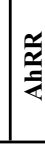 & 光 & & 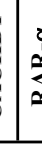 & 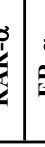 & & ئ & 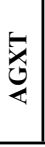 & $\bar{E}$ & 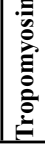 & 商 & $\hat{\hat{\hat{\theta}}}$ & $\frac{3}{2}$ & 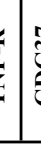 & 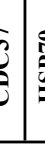 & 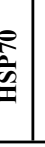 & $\xi$ & 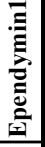 & 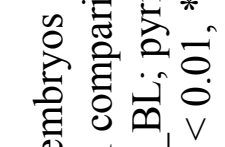 \\
\hline ثิ & & & & & & & & & r & & & & & & & & & & & & & & $E *$ \\
\hline$\dot{z}$ & - & N & $m$ & + & in & & & $\alpha$ & 。 & & & & $\simeq$ & $=$ & \pm & 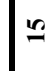 & 5 & $:$ & $=1$ & $\infty$ & & ते & 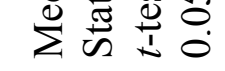 \\
\hline
\end{tabular}


The expression of $A h R 2 a$ was enhanced by 1.5 fold upon exposure to $380^{\circ} \mathrm{C}$ thermally treated pyridinol. In contrast, exposure to $300^{\circ} \mathrm{C}$ thermally treated chlorpyrifos induced $A h R 2 a$ more than $2.5-3.0$ fold, but suppressed it at higher thermal treatment, whereas $A h R 1 b-1$ was slightly induced by exposure to either chlorpyrifos or pyridinol.

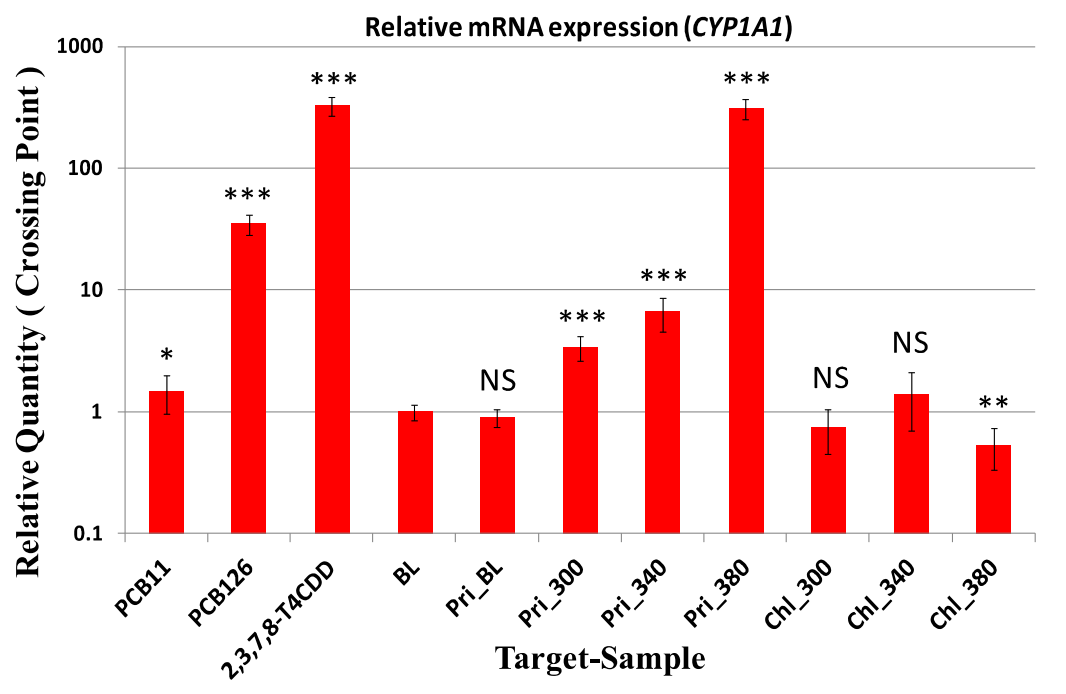

Fig. 1 - CYP1A1 mRNA expression in medaka embryos exposed to pyrolysates of chlorpyrifos and 3,5,6-trichloro-2-pyridinol. The amount of CYP1A1 mRNA was measured by quantitative real-time PCR and normalized to the solvent control $(0.1 \%$ DMSO) for $48 \mathrm{~h}$ exposure, the error bars represents SD $(\mathrm{n}=3)$. BL; Blank (DMSO 0.1\%), Pri_BL; pyridinol-blank (non-thermal treatment), Pri; pyridinol, Chl; chlorpyrifos, _300; pyrolysates under $300^{\circ} \mathrm{C}$ treatment. ${ }^{*} \mathrm{p}<0.05,{ }^{* *} \mathrm{p}<0.01,{ }^{* * *} \mathrm{p}<0.00 \overline{1}, \mathrm{NS}$; not significant $(\mathrm{p}>0.05)$.

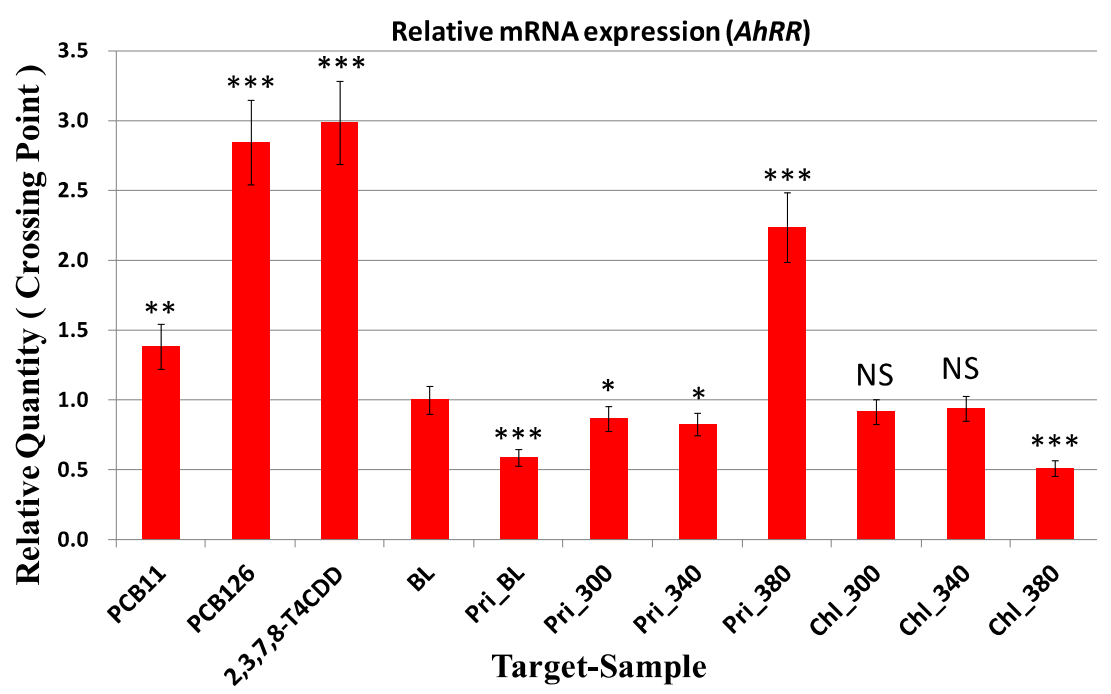

Fig. 2 - AhRR mRNA expression in medaka embryos exposed to pyrolysates of chlorpyrifos and 3,5,6-trichloro-2-pyridinol. 


\section{Group2 genes}

The functions of the Group2 genes were endocrine and reproductive effects. Induction of CACHD1 (Cache domain-containing protein 1) mRNA expression is shown in Fig. 4. CACHD1 contains two cache domains and a VWFA (von Willebrand factor A) domain which is known to be involved in homeostasis (Anantharaman and Aravind, 2000). This gene was induced by the exposure to heavy metals or PCBs with low TEF, but was suppressed by PCDDs with high TEF (e.g. 2,3,7,8-T4CDD). The expression of $C A C H D 1$ mRNA was enhanced by pyridinol exposure, but was suppressed by chlorpyrifos exposure at higher thermal treatment. The mRNA expression of $R A R-\alpha$ and $E R-\alpha$ also showed similar patterns as $C A C H D 1$ at thermal treatment.

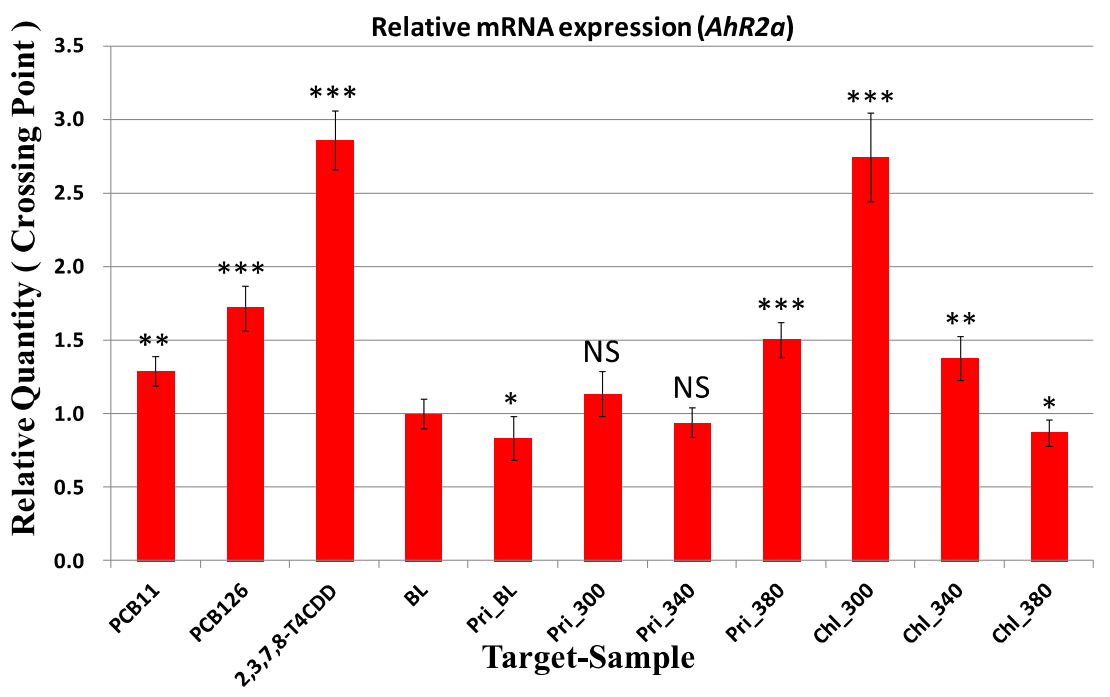

Fig. 3 - AhR2a mRNA expression in medaka embryos exposed to pyrolysates of chlorpyrifos and 3,5,6-trichloro-2-pyridinol.

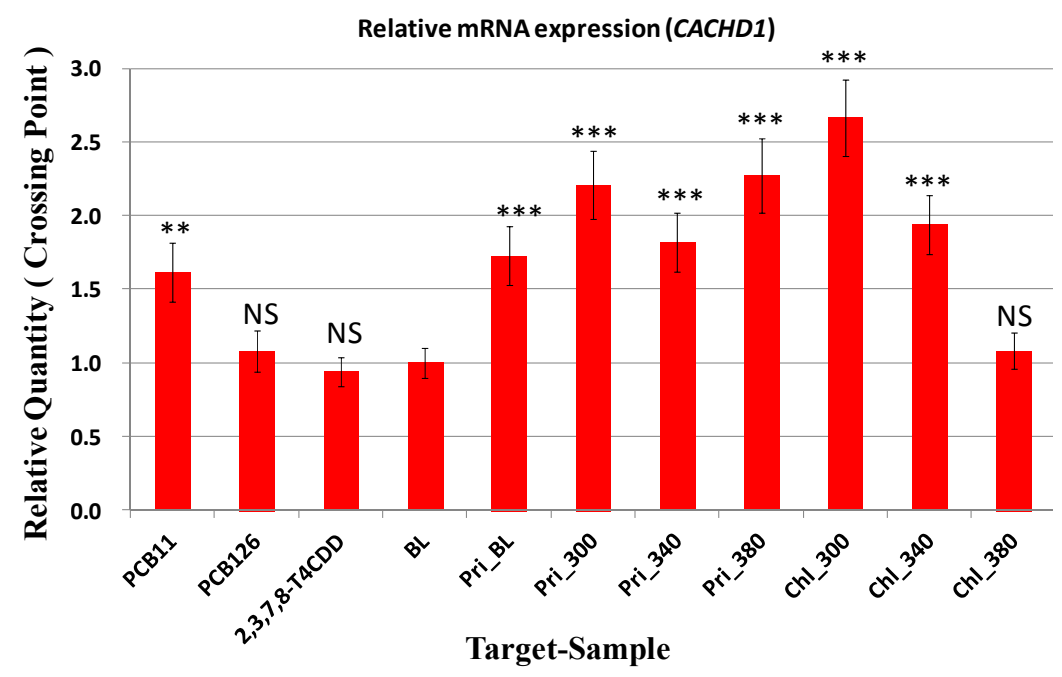

Fig. 4 - CACHD1 mRNA expression in medaka embryos exposed to pyrolysates of chlorpyrifos and 3,5,6-trichloro-2-pyridinol. 


\section{Group3 genes}

The function of the Group3 genes was cell proliferation. Induction of HSP90 mRNA expression is shown in Fig. 5. Heat shock proteins (HSPs) are stress-defensive proteins and are highly induced in response to stresses caused by changes in environmental factors such as high temperature, heavy metal exposure, reactive oxygen production, salinity, etc (Beckmann et al., 1990) and protect the structure and function of proteins and cells from damages, maintaining the cellular homeostasis. HSP90 mRNA expression was suppressed by exposure to both pyridinol and chlorpyrifos.

\section{Group4 genes}

The functions of the Group4 genes were immunity effects and nervous system control. Induction of Metallothionein (MT) mRNA expression is shown in Fig. 6. MT is a

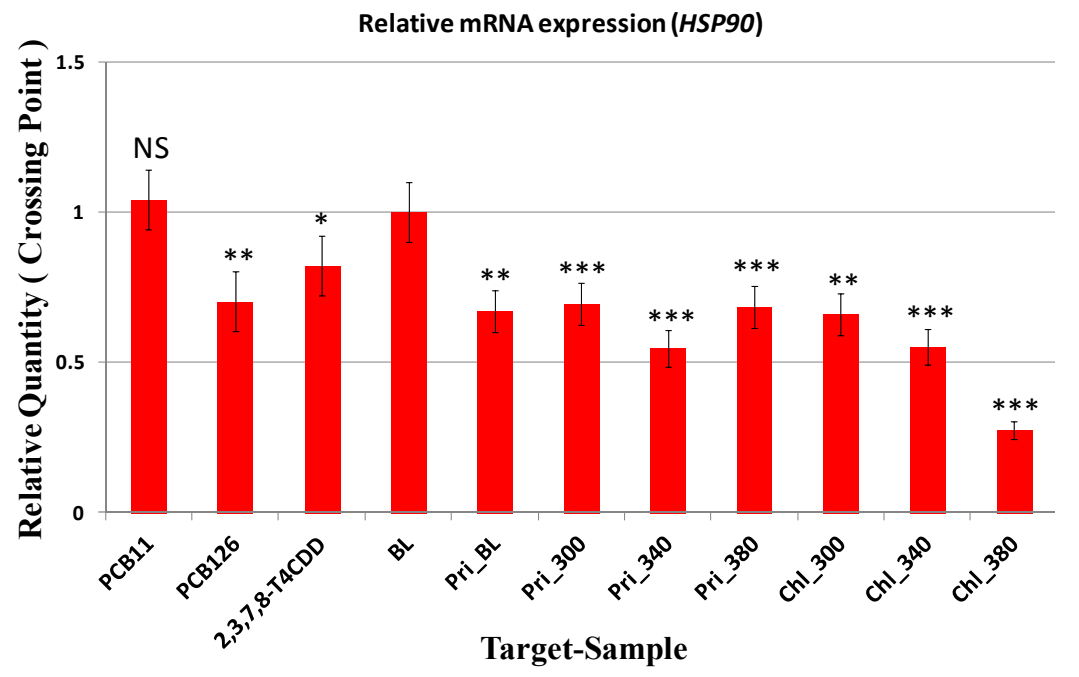

Fig. 5 - HSP90 mRNA expression in medaka embryos exposed to pyrolysates of chlorpyrifos and 3,5,6-trichloro-2-pyridinol.

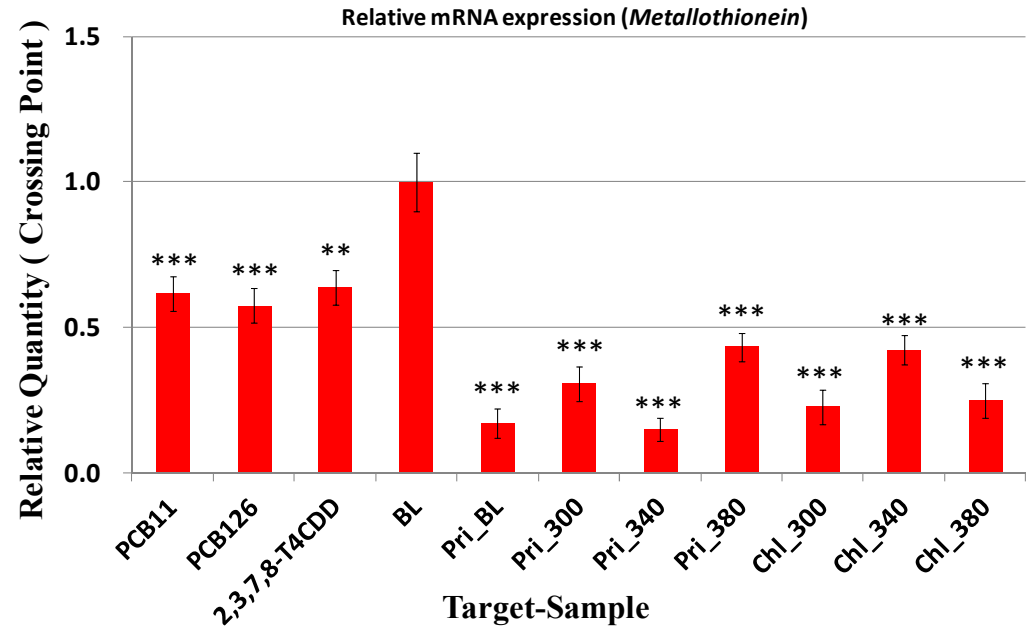

Fig. 6 - MT mRNA expression in medaka embryos exposed to pyrolysates of chlorpyrifos and 3,5,6-trichloro-2-pyridinol. 
protein that has high cysteine content, low molecular mass (about $7 \mathrm{kDa}$ ), and high affinity for metals. It binds metals and regulates the homeostasis of essential trace metals such as copper and zinc, and also takes a part in counteracting the toxic effects of heavy metals such as cadmium, mercury, and silver (Choi et al., 2007). The mRNA expressions of $M T$ were suppressed by exposure to both pyridinol and chlorpyrifos. The same expression patterns were shown by HSP70 and TBP.

\section{Toxicity assessment of thermal treatments of chlorpyrifos and pyridinol}

The pyrolysate of pyridinol $(1.3 \mathrm{mg} / \mathrm{L})$ after $380^{\circ} \mathrm{C}$ thermal treatment induced CYP1A1, $A h R 2 a, A h R R$ (Group1) and CACHD1, RAR- $\alpha$ (Group2), but suppressed HSP90 (Group3) and MT (Group4). 2,3,7,8-T4CDD (1 $\mu \mathrm{g} / \mathrm{L})$ induced CYP1A1, AhR2a, and AhRR (Group1), but suppressed HSP90 (Group3), HSP70 and MT (Group4).

In this study, we investigated the adverse effects of the pyrolysate of pyridinol (1.3 $\mathrm{mg} / \mathrm{L}$ ) with $380^{\circ} \mathrm{C}$ thermal treatment on the occurrence of developmental abnormalities in medaka embryos by observation with a microscope. The pyrolysate caused blood circulation deficiencies, including slowed blood flow, blood clots, and hemorrhage, in the caudal vein and arteries the same as 2,3,7,8-T4CDD $(1 \mu \mathrm{g} / \mathrm{L})$. These results suggest that the thermal treatment at $380^{\circ} \mathrm{C}$ of pyridinol might form the pyridine analogue of 2,3,7,8-T4CDD which has the very similar ecotoxicities to 2,3,7,8-T4CDD in medaka embryos. In contrast, the pyrolysates of chlorpyrifos did not induce CYP1A1 and AhRR, which were well known to be sensitive target genes for dioxins and dioxin-like compounds.

The formation potential of the pyridine analogue of 2,3,7,8-T4CDD was considerably lower for chlorpyrifos compared to pyridinol, indicating that the phosphoric acid ester moiety might considerably reduce the dioxin-precursor potential of the compounds (Fig. 7). Sakiyama et al. (2011) confirmed that the yield of the pyridine analogue of 2,3,7,8-T4CDD was considerably higher in the pyrolysate of pyridinol than that of chlorpyrifos by GC/MS analysis. Wheelock et al. (2005) studied chlorpyrifos and clarified that chlorpyrifos had little effect on CYP1A protein levels in salmon, suggesting OPs (organophosphates) may not be strong regulators of CYP1A in fish. The main effect of OPs on CYP1A appears to be catalytic suppression (Flammarion et al., 1996; Paolini et al., 1997; Flammarion et al., 1998; Tang et al., 2002). Organophosphate effects on CYPs occur as a result of CYP-mediated metabolism, where OPs, such as chlorpyrifos, undergo desulfuration by CYPs leading to the release
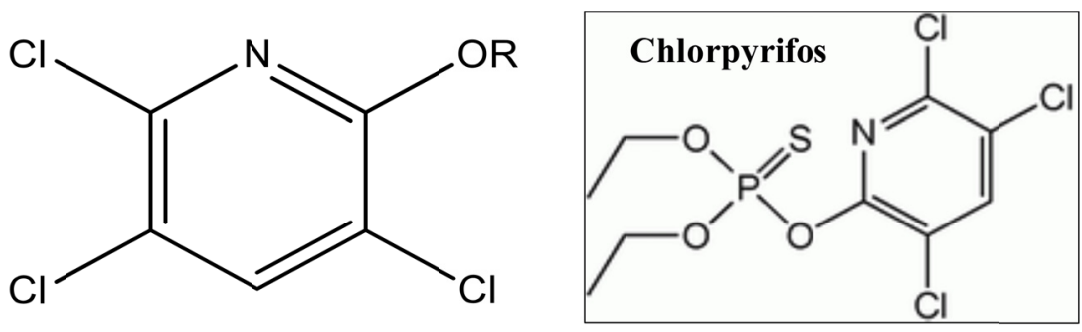

\section{$\mathbf{R}=\mathbf{H}:$ 3,5,6-trichloro-2-pyridinol (a potential precursor)}

$\mathbf{R}=$ Phosphoric acid ester :Chlorpyrifos

Fig. 7 - Structures of 3,5,6-trichloro-2-pyridinol and chlorpyrifos. 
of a free sulfur ion, which binds to the CYP heme and inhibits catalytic activity (Fukuto, 1990; Tang et al., 2002). These results suggest that the pyrolysates of chlorpyrifos would contain the unknown ecotoxic chemicals other than the predicted pyridine analogue of 2,3,7,8-T4CDD. Further assessment is necessary to clarify the potential ecotoxicities of chlorpyrifos and its analogues and the advantages of thermal treatment on these chemicals to reduce their ecotoxicities.

\section{CONCLUSIONS}

In this study, we evaluated the toxicities of the pyrolysates formed in thermal treatments of pyridinol and chlorpyrifos at $300,340,380^{\circ} \mathrm{C}$ for 10 minutes, obtaining the following conclusions :

1. The pyridine analogue of 2,3,7,8-T4CDD would be formed in thermal treatment of pyridinol which possesses the very similar ecotoxicities to $2,3,7,8$-T4CDD.

2. The formation potential of the pyridine analogue of 2,3,7,8-T4CDD was considerably lower for chlorpyrifos compared to pyridinol, indicating that the phosphoric acid ester moiety might considerably reduce the dioxin-precursor potential.

The pyridine analogue of 2,3,7,8-T4CDD may also be formed and released when chlorpyrifos-impacted crop residues and biomass are burned and when chlorpyrifos-contaminated soil is heated. These findings need further assessment considering that chlorpyrifos is a major pesticide used worldwide and can be involved in fires in pesticide productions and storages and in the combustion of post-harvest residues with associated thermal stress for present dioxin and dioxin-like substance precursors. Further assessment on chlorpyrifos at higher temperature thermal treatment is therefore needed to assess the potentialities of the ecotoxic chemical formation.

\section{REFERENCES}

Anantharaman V. and Aravind L. (2000) Cache - a signaling domain common to animal $\mathrm{Ca}^{2+}$-channel subunits and a class of prokaryotic chemotaxis receptors. Trends Biochem. Sci., 25(11), 535-537.

Beckmann R. P., Mizzen L. E. and Welch W. J. (1990) Interaction of Hsp 70 with newly synthesized proteins: implications for protein folding and assembly. Science, 248, 850-854.

Choi C. Y., An K. W., Nelson E. R. and Habibi H. R. (2007) Cadmium affects the expression of metallothionein (MT) and glutathione peroxidase (GPX) mRNA in goldfish, Carassius auratus. Comp. Biochem. Physiol., C145(4), 595-600.

Flammarion P., Migeon B. and Garric J. (1996) Joint effects of copper sulphate and methidathion on rainbow trout (Oncorhynchus mykiss) EROD and AChE activities. Bull. Environ. Contam. Toxicol., 56(3), 440-445.

Flammarion P., Migeon B., Urios S. B., Morfin P. and Garric J. (1998) Effect of methidathion on the cytochrome P-450 1A in the cyprinid fish gudgeon (Gobio gobio). Aquatic Toxicol., 42(2), 93-102.

Fukuto T. R. (1990) Mechanism of action of organophosphorus and carbamate insecticides. Environ. Health Persp., 87(2), 245-254. 
Hagenmaier H. and Berchtold A. (1986) Analysis of waste from production of Na-pentachlorophenolate for polychlorinated dibenzodioxins (PCDD) and dibenzofurans (PCDF). Chemosphere, 15(9-12), 1991-1994.

Hahn M. E., Allan L. L. and Sherr D. H. (2009) Regulation of constitutive and inducible AHR signaling: Complex interactions involving the AHR repressor. Biochem. Pharmacol., 77(4), 485-497.

Hanno K., Oda S. and Mitani H. (2010) Effects of dioxin isomers on induction of $A h R s$ and CYPIA1 in early developmental stage embryos of medaka (Oryzias latipes). Chemosphere, 78(7), 830-839.

Holt E., Weber R., Stevenson G. and Gaus C. (2010) Polychlorinated dibenzo- $p$-dioxins and dibenzofurans (PCDD/Fs) impurities in pesticides: A neglected source of contemporary relevance. Environ. Sci. Technol., 44(14), 5409-5415.

Livak K. J. and Schmittgen T. D. (2001) Analysis of relative gene expression data using real-time quantitative PCR and the $2^{-\Delta \Delta C_{\mathrm{T}}}$ method. Methods, 25(4), 402-408.

Masunaga S., Takasuga T. and Nakanishi J. (2001) Dioxin and dioxin-like PCB impurities in some Japanese agrochemical formulations. Chemosphere, 44(4), 873-885.

Mocarelli P. (2001) Seveso: a teaching story. Chemosphere, 43(4-7), 391-402.

Paolini M., Pozzetti L., Sapone A., Mesirca R., Perocco P., Mazzullo M. and Cantelli-Forti G. (1997) Molecular non-genetic biomarkers of effect related to acephate cocarcinogenesis: sex- and tissue-dependent induction or suppression of murine CYPs. Cancer Lett., 117(1), 7-15.

Sakiyama T., Weber R., Behnisch P. and Nakano T. (2011) Preliminary assessment of dioxin-like compounds in/from chlorpyrifos-A potential precursor of the pyridine analogue of 2,3,7,8-TCDD. International Symposium on Halogenated Environmental Organic Pollutants and POPs, Dioxin 2011, Brussels, Belgium.

Tang J., Cao Y., Rose R. L. and Hodgson E. (2002) In vitro metabolism of carbaryl by human cytochrome P450 and its inhibition by chlorpyrifos. Chem. Biol. Interact., 141(3), 229-241.

Weber R., Gaus C., Tysklind M., Johnston P., Forter M., Hollert H., Heinisch E., Holoubek I., Lloyd-Smith M., Masunaga S., Moccarelli P., Santillo D., Seike N., Symons R., Torres J., Verta M., Varbelow G., Vijgen J., Watson A., Costner P., Woelz J., Wycisk P. and Zennegg M. (2008) Dioxin- and POP-contaminated sites -contemporary and future relevance and challenges. Environ. Sci. Poll. Res., 15(5), 363-393.

Wheelock C. E., Eder K. J., Werner I., Huang H., Jones P. D., Brammell B. F., Elskus A. A. and Hammock B. D. (2005) Individual valiability in esterase activity and CYP1A levels in Chinook salmon (Oncorhynchus tshawytscha) exposed to esfenvalerate and chlorpyrifos. Aquatic Toxicol., 74(2), 172-192. 